\title{
A review of traction battery model and parameter identification in electric vehicle
}

\author{
Chunyang Wang ${ }^{1}$, Bo Xing ${ }^{1}$, Jiaping Zhou ${ }^{1}$ \\ ${ }^{1}$ Beijing CATARC Technologu Co., Ltd., China Automotive Technology \& Research Center, F/9, 2-7 Building ABP, 188Western Rd., \\ South 4th Ring Rd., Fengtai District, Beijing, 100070, China
}

\begin{abstract}
State estimation is a key issue of battery management system (BMS) to improve the energy utilization of traction battery in electric vehicle, which is usually achieved based on battery model. The commonly used models, equivalent circuit model (ECM) and electrochemical mechanism-based model (EMM), are reviewed in this paper. Besides, the corresponding parameter identification methods are analysed considering the target application background.
\end{abstract}

\section{Introduction}

As the important means of transportation, vehicle plays an important role in our daily life. With the increasing of vehicle holdings, the vehicles with oil as the main fuel source have brought a lot of pressure to the energy and environment all over the world. Under this circumstance, the new energy vehicle has become the new development direction of auto industry, among which electric vehicle is the main force.

Due to the high energy density and long lifetime, Lithium-ion batteries are wildly applied as the in-vehicle energy storage unit 12 . Safe and efficient management of lithium-ion battery is the key to take full advantage of battery energy and extend the driving range of electric vehicles. The structure and working principle of traction battery system in electric vehicle are shown in Fig. 1.

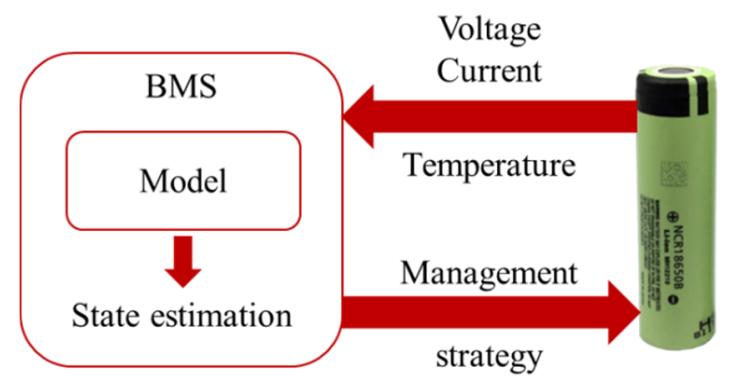

Fig. 1. Structure and work principle of traction battery system in electric vehicle
In general, battery states are estimated based on model as well as the sampling working conditions and are used as the basis of developing management strategy in battery management system (BMS). Therefore, the model embedded in the BMS should provide high accuracy and real-time performance. Meanwhile, the applicable methods of parameter identification need to be chosen according to the application purpose and conditions.

\section{Lithium-ion battery model and parameter identification}

The widely used models of battery state estimation include equivalent circuit model (ECM) and electrochemical mechanism-based model (EMM).

\subsection{Equivalent circuit model}

The ECM mainly simulates lithium-ion battery external characteristics by building equivalent circuit with electronic components such as resistor and capacitor. The earliest R-int model just reflects battery inner impedance with a resistor, and a single RC module in parallel is added to describe electrode polarization in Thevenin model 3. Hu 4 analyzed 12 ECM including models with $\mathrm{nRC}$ module or hysteresis module and found that the higher order of the model leads to the higher accuracy with more calculation added inevitably. 


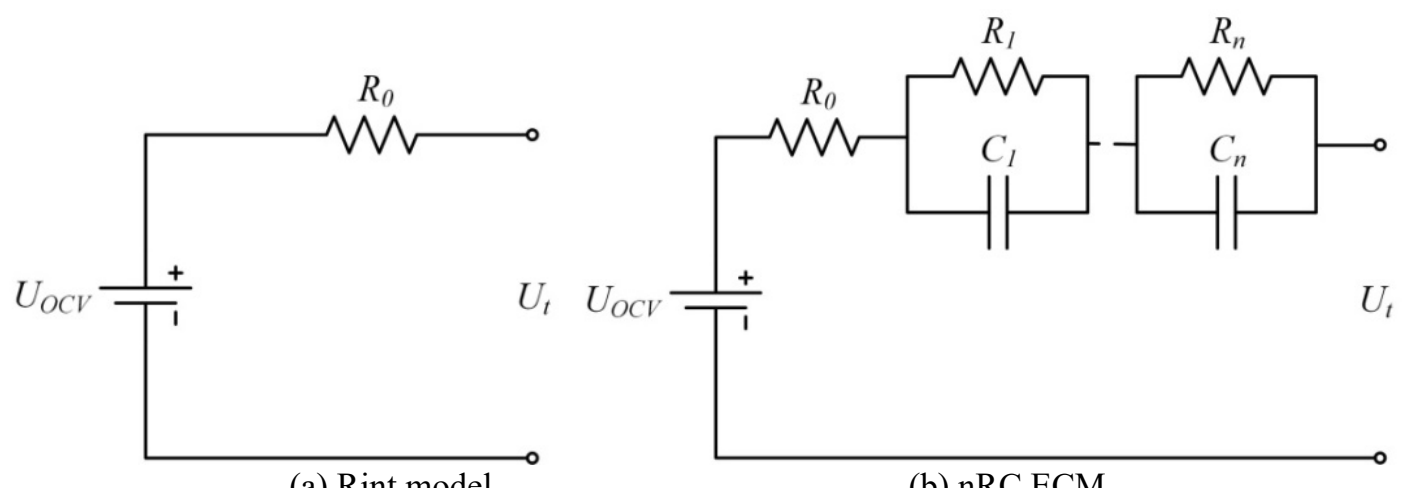

(a) Rint model

(b) nRC ECM

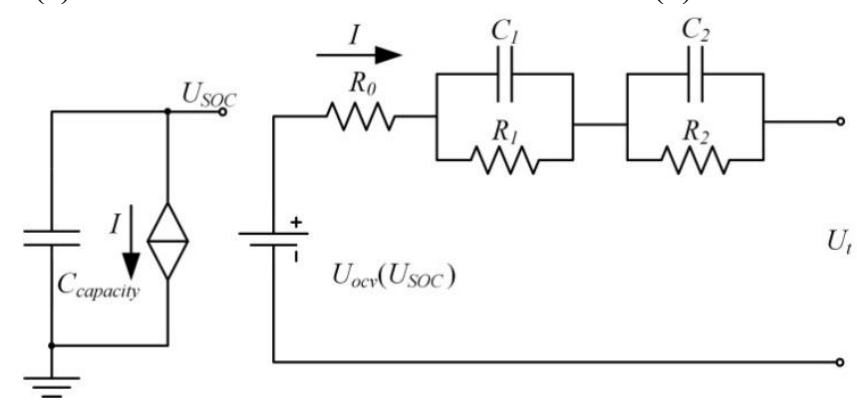

(c) Runtime-based model

Fig. 2. The commonly used ECM

The runtime-based model (RTM) was established with SOC simulation module added to the second-order RC ECM. In the RTM, current-controlled current source was used for battery working current simulation and the real-time SOC was obtained by ampere-hour integral567. Both the terminal voltage response and battery runtime estimation could be carried out, but there wasn't essential difference in voltage simulation compared to the secondorder RC ECM.

\subsection{Electrochemical mechanism-based model}

The EMM simulates the working characteristics of lithium-ion battery by describing the internal electrochemical reactions. Pseudo-two-dimensional (P2D) model 8, based on porous electrode theory and concentrated solution theory, is regarded as the basis of EMM. With a series of partial differential equations employed to precisely describe the internal electrochemical mechanism, P2D model is capable to simulate both external characteristics of lithium-ion battery and the variation of inner electrochemical variables 910 , but the extremely complex computation makes it difficult to operate in real-time 1112.

To achieve the dual effect of simplifying model operations while maintaining model accuracy, some simplified EMMs have been developed, among which, the single particle model (SPM) is the most representative 13. With the assumption that the electrochemical reactions at the electrodes are uniform, the active material of each electrode is simplified to a single particle and the electrolyte phase diffusion is neglected. Accordingly, the simulation accuracy of the SPM at high-rate is lost 1415. Besides, EMM involves many electrochemical parameters, the identification of which is usually time-consuming and can hardly be achieved online 16 .

\section{Model parameter identification}

Parameter identification of the established model makes a great impact on model simulating performance. In general, the identification methods are mainly divided into online identification and offline identification.

\subsection{Online identification}

Online identification helping model parameters to update in real time is achieved though algorithms such as Kalman Filter (KF), recursive least square method, and $\mathrm{H}$ infinity algorithm 17, in which the sampling data works as the feedback 18. Thus, online parameter estimation is beneficial to adapt the model to dynamic working conditions.

\subsection{Offline identification}

Offline identification of parameters is usually realized by state equation fitting or genetic algorithm based on the battery experimental data under specific working conditions 1920. Although the precision of parameters is not as good as those identified online, offline identification shows advantages from some aspects. On one hand, the real-time operation efficiency of the model could be improved with the parameters calibrated offline. On the other hand, it offers solution to the cases where online identification could not be realized. For instance, the parameters of the EMM are highly coupled 2122 and the parameter identification in the prediction time domain lacks of real-time systematic feedback 23 . 


\section{Conclusion}

As for model embedded in the BMS, the ECM is more suitable for battery real-time state estimation in electric vehicle, balancing the calculation and estimation accuracy. And the limitation of the ECM in simulation accuracy at low SOC range could be got over by combining the internal electrochemical process of lithium-ion battery.

In the above model parameter identification methods, the offline identification is not adaptable to dynamic conditions, and the online identification causes initial errors and could hardly be achieved in the prediction period. Thereby, online identification is suitable for updating parameters in real time based on output error, while offline identification could be used to obtain parameters in typical battery state, to correct possible initial errors in online identification, and to calibrate model parameters for state prediction.

\section{References}

1. Lu L, Han X, Li J, Hua J, Ouyang M. A review on the key issues for lithium-ion battery management in electric vehicles. J Power Sources 2013; 226:272-88.

2. Liu K, Li K, Peng Q, Zhang C. A brief review on key technologies in the battery management system of electric vehicles. Front Mech Eng 2019; 14:47-64.

3. Coleman M, Hurley WG, Lee CK. An improved battery characterization method using a two-pulse load test. IEEE Trans Energy Convers 2008; 23: 708-13.

4. $\mathrm{Hu} \mathrm{X}, \mathrm{Li} \mathrm{S}$, Peng $\mathrm{H}$. A comparative study of equivalent circuit models for Li-ion batteries. J Power Sources 2012; 198:359-67.

5. Benini L, Castelli G, Macii A, Macii E, Poncino M, Scarsi R. Discrete-time battery models for systemlevel low-power design. IEEE Trans Very Large Scale Integr Syst 2001; 9:630-40.

6. Chen M, Rincón-Mora GA. Accurate electrical battery model capable of predicting runtime and I-V performance. IEEE Trans Energy Convers 2006;21: 504-11.

7. Sarrafan K, Sutanto D, Muttaqi KM. An electric circuit based EV battery model for runtime prediction and state of charge tracking. 2017 IEEE Transp Electrif Conf ITEC-India 2017 2018;2018Janua:1-6.

8. Doyle M. Comparison of Modeling Predictions with Experimental Data from Plastic Lithium Ion Cells. J Electrochem Soc 1996.

9. Zheng L, Zhang L, Zhu J, Wang G, Jiang J. Coestimation of state-of-charge, capacity and resistance for lithium-ion batteries based on a high-fidelity electrochemical model. Appl Energy 2016.

10. Xiong R, Li L, Li Z, Yu Q, Mu H. An electrochemical model based degradation state identification method of Lithium-ion battery for allclimate electric vehicles application. Appl Energy
2018; 219: 264-75.

11. Nejad S, Gladwin DT, Stone DA. A systematic review of lumped-parameter equivalent circuit models for real-time estimation of lithium-ion battery states. J Power Sources 2016.

12. Bi Y, Choe SY. An adaptive sigma-point Kalman filter with state equality constraints for online stateof-charge estimation of a $\mathrm{Li}(\mathrm{NiMnCo}) \mathrm{O} 2 / \mathrm{Carbon}$ battery using a reduced-order electrochemical model. Appl Energy 2020.

13. Rahimian SK, Rayman S, White RE. Comparison of single particle and equivalent circuit analog models for a lithium-ion cell. J Power Sources 2011; 196: 8450-62.

14. Santhanagopalan S, Guo Q, Ramadass P, White RE. Review of models for predicting the cycling performance of lithium ion batteries. $\mathrm{J}$ Power Sources 2006; 156: 620-8.

15. Li J, Wang D, Pecht M. An electrochemical model for high C-rate conditions in lithium-ion batteries. J Power Sources 2019; 436:226885.

16. Li J, Wang L, Lyu C, Wang D, Pecht M. Parameter updating method of a simplified first principlesthermal coupling model for lithium-ion batteries. Appl Energy 2019.

17. Yu Q, Xiong R, Lin C, et al. Lithium-ion Battery Parameters and State-of-Charge Joint Estimation Based on $\mathrm{H}$ infinity and Unscented Kalman Filters[J]. IEEE Transactions on Vehicular Technology, 2017, PP (99):1-1.

18. Zhang J, Wei Y, Li X, et al. Battery SOC estimation based on online parameter identification[J]. Diangong Jishu Xuebao/transactions of China Electrotechnical Society, 2014, 29:23-28.

19. Xiong R, Core algorithm of battery management system for EVs, China Machine Press, 2018.

20. Forman JC, Moura SJ, Stein JL, Fathy HK. Genetic identification and fisher identifiability analysis of the Doyle-Fuller-Newman model from experimental cycling of a LiFePO 4 cell. J Power Sources 2012; 210:263-75.

21. Luo W, Lyu C, Wang L, Zhang L. A new extension of physics-based single particle model for higher charge-discharge rates. J Power Sources 2013; 241:295-310.

22. Li J, Wang L, Lyu C, Liu E, Xing Y, Pecht M. A parameter estimation method for a simplified electrochemical model for Li-ion batteries. Electrochim Acta 2018; 275:50-8.

23. Liu G, Ouyang M, Lu L, Li J, Hua J. A highly accurate predictive-adaptive method for lithium-ion battery remaining discharge energy prediction in electric vehicle applications. Appl Energy 2015;149: 297-314. 